\title{
Stem cell therapy: social recognition recovery in a FASD model
}

\author{
T Shirasaka, E Hashimoto, W Ukai, T Yoshinaga, T Ishii, M Tateno and T Saito
}

To better understand the cellular pathogenetic mechanisms of fetal alcohol spectrum disorder (FASD) and the therapeutic benefit of stem cell treatment, we exposed pregnant rats to ethanol followed by intravenous administration of neural stem cells (NSCs) complexed with atelocollagen to the new born rats and studied recovery of GABAergic interneuron numbers and synaptic protein density in the anterior cingulate cortex, hippocampus and amygdala. Prenatal ethanol exposure reduced both parvalbumin-positive phenotype of GABAergic interneurons and postsynaptic density protein 95 levels in these areas. Intravenous NSC treatment reversed these reductions. Furthermore, treatment with NSCs reversed impaired memory/cognitive function and social interaction behavior. These experiments underscore an important role for synaptic remodeling and GABAergic interneuron genesis in the pathophysiology and treatment of FASD and highlight the therapeutic potential for intravenous NSC administration in FASD utilizing atelocollagen.

Translational Psychiatry (2012) 2, e188; doi:10.1038/tp.2012.111; published online 13 November 2012

\section{Introduction}

Prenatal alcohol exposure is invariably detrimental to the developing central nervous system and can cause behavioral/ cognitive and mental/social problems, defined as fetal alcohol spectrum disorders (FASDs). ${ }^{1,2}$ Abnormalities in cortical and limbic system development have been linked to FASD, but the underlying cellular pathogenesis in the brain remains elusive. The cortex, hippocampus and amygdala, which are key structures in memory and emotional/social behaviors, ${ }^{3-5}$ are brain regions that have been implicated in the pathophysiology of FASD, Dysfunction of appropriate GABAergic inhibition and the consequent imbalance between excitation and inhibition in the corticolimbic neural network underlies at least part of the pathophysiological process of several neuropsychiatric disorders, including schizophrenia, autism and FASD. $^{6-8}$ A number of studies have found alterations in several presynaptic and postsynaptic components of the GABAergic systems, especially in reduced levels of the rate-limiting synthetic enzyme glutamic-acid-decarboxylase 67 (GAD67) as observed in human and animal samples of schizophrenia and FASD. ${ }^{9,10}$ In addition, in schizophrenia parvalbumin (PV)-positive interneurons, many of which display a fast-spiking firing pattern, were severely disrupted. ${ }^{11,12}$ As the ability of PV-containing fast-spiking interneurons to drive synchronous oscillatory activity at gamma-frequency is being acknowledged as a cellular basis for cognitive and executive brain function, it follows that PV interneuron dysfunction could induce social/cognitive behavioral abnormalities. ${ }^{13}$ What type of GABAergic cell development was specifically influenced by prenatal ethanol exposure is unknown; however, the behavioral abnormalities observed in children affected with FASD include cognitive, executive and social/emotional dysfunctions, which are indicative of disruption in corticolimbic (including PV-containing) interneurons.

Stem cell-based regenerative therapy promises great benefits for patients with incurable brain diseases. Previous studies have shown that stem cell treatments improve behavioral deficits in animal models of neurological disorders such as Parkinson's, Huntington's and Alzheimer's diseases. Stem cell therapy involves two possible strategies: cell replacement and cell restoration. The administered stem cells substitute for the lost cells in a disease and/or provide a trophic effect, protect, rescue and minimize the deteriorating tissue affected in a disease. Although ethical and technical issues remain unresolved, stem cell therapy would proceed from neurological to psychiatric illness such as affective disorders and schizophrenia, after having tried all the available medications and electroconvulsive therapy.

We studied the involvement of corticolimbic GABAergic interneuron disruption in cognitive and social impairment in FASD and the effect of stem cell treatment. We used an animal model of FASD created in fetal rats by the binge-like administration of ethanol during the peak of GABAergic cell generation of dams (E11-E14). ${ }^{10}$ We show here that aspects of cognitive and social dysfunction are reversed by intravenous administration of fetal rat brain-derived neural stem cells (NSCs) with atelocollagen, used to reduce immune rejection and potentiate effective migration of administrated cells into brain, at 45 days, a time point when neural developments are already completed. We also show that alterations of PVcontaining GABAergic interneurons and synaptic density protein levels are essential for the therapeutic efficacy of intravenous NSC treatment in this animal model. Our results

Department of Neuropsychiatry, School of Medicine, Sapporo Medical University, Chuo-ku, Sapporo, Japan

Correspondence: Dr T Shirasaka, Department of Neuropsychiatry, School of Medicine, Sapporo Medical University, S-1, W-16, Chuo-ku, Sapporo, 0608543, Japan. E-mail: shirasaka.t@sapmed.ac.jp

Keywords: atelocollagen; cell therapy; fetal alcohol spectrum disorder; GABAergic interneuron; neural stem cell

Received 24 July 2012; revised 6 September 2012; accepted 6 September 2012 
establish a new role for PV-containing GABAergic interneurons in the behavioral abnormalities of cognitive and social dysfunction associated with FASD and suggest new therapeutic mechanisms for stem cell treatment making use of the hydrogel scaffold, atelocollagen.

\section{Materials and methods}

Materials. The following materials were purchased: Hank's balanced salt solution, hneurobasal medium, B27 and 5-(and-6)-carboxyfluorescein diacetate succinimidyl ester (CFSE) from Invitrogen (Carlsbad, CA, USA), recombinant human fibroblast growth factor-2 from PeproTech (London, UK), cyclosporine from Novartis Pharma (Tokyo, Japan) and atelocollagen from Koken (Tokyo, Japan).

Experimental design and prenatal ethanol treatment. Pregnant Wistar rats were purchased from Clea Japan (Sapporo, Japan). Rats were administered ethanol $\left(3 \mathrm{~g} \mathrm{~kg}^{-1}\right.$, estimated mean blood concentration: $150 \mathrm{mg} \mathrm{dl}^{-1}$ ) or an equivalent volume of physiological saline via an intragastric catheter every $12 \mathrm{~h}$ for 4 days on gestational days $10-13$ as described previously. ${ }^{14}$ Each rat was housed in an individual cage until delivery and was allowed to give birth. The pups were fed by their biological lactating mothers and weaned at 30 days. Rats were housed at $22^{\circ} \mathrm{C}$ on a 12:12-h light-dark cycle with free access to food and water. All experimental procedures were approved by the institutional animal care committee and conducted following the Sapporo Medical University Guidelines for the Care and Use of Laboratory Animals. The experimental design is shown in Figure 1a. To study the effect of NSC therapy on abnormal behavior dependent on memory and cognitive dysfunction, rats were subjected to ethanol treatment for 4 consecutive days at gestational days 10-13, followed by an intravenous administration of fetal rat brain-derived NSCs at postnatal day $(P)$ 45. The administrated NSCs were pre-mixed with $0.2 \%$ atelocollagen to avoid immune rejection and to facilitate migration into the brain. At 90 days after the administration of NSCs, controls and alcohol exposed rats were tested for their memory, cognitive and social recognition behavior. The rats were divided into four groups: (1) controls: rats received saline on P45 with prenatal saline exposure. (2) FASD: rats were given saline on P45 with prenatal ethanol exposure. (3) FASD + NSC: rats received an NSC injection at P45 with prenatal ethanol exposure. (4) Control + NSC: rats were given NSC injection at P45 with prenatal saline exposure. Three month after administration of NSCs or saline, we tested behavioral memory and social recognition followed by pathological analysis of brain samples.

NSC preparation and labeling. NSCs were obtained from 13.5-day-old rat embryos and cultured in a monolayer as previously described. ${ }^{15}$ Briefly, telencephalic neuroepithelium
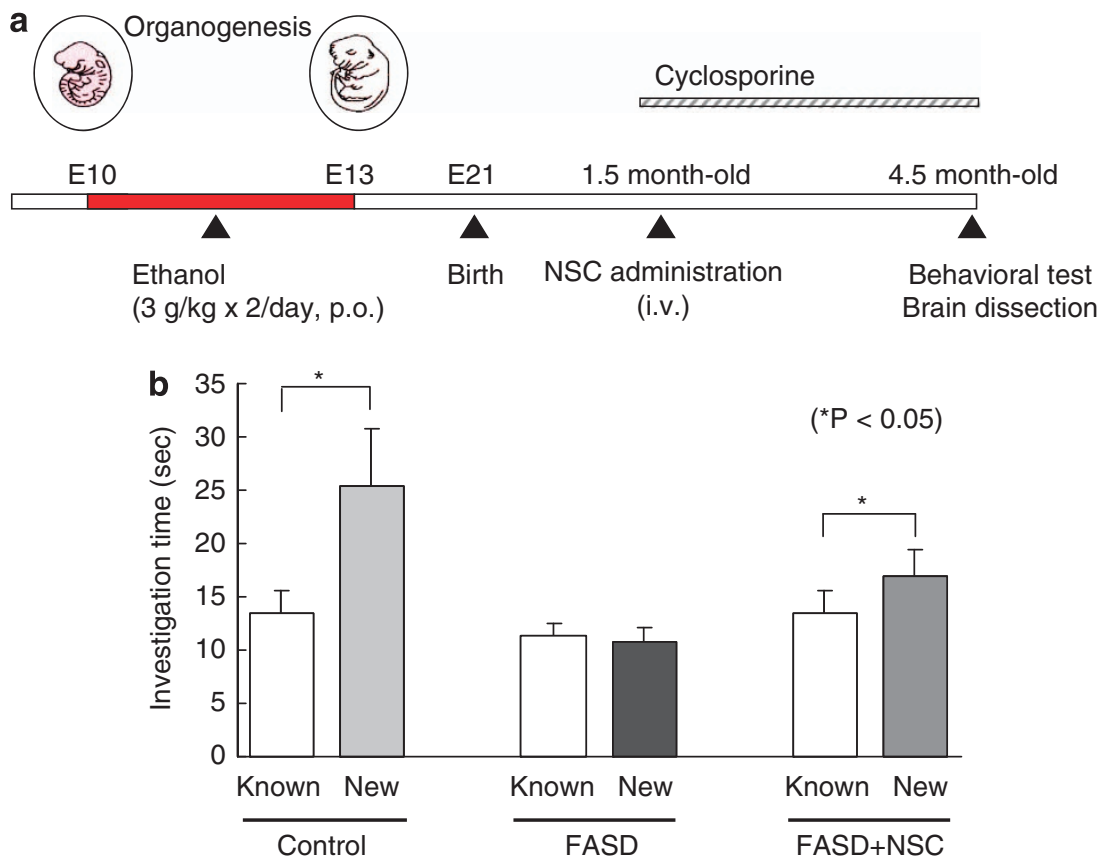

Figure 1 Experimental design and memory and recognition test. (a) Experimental schedule of neural stem cell (NSC) administration to fetal alcohol spectrum disorder (FASD) model rat. FASD model rat was obtained by oral treatment of ethanol $\left(3 \mathrm{~g} \mathrm{~kg}^{-1} \times 2\right.$ per day) during embryonic day (E) $10-13$ of the pregnancy. Carboxyfluorescein diacetate succinimidyl ester (CFSE) fluorescent-labeled NSC solution mixed with atelocollagen was injected intravenously into 45-day-old newborn rats of control or FASD groups. Control, control + NSC, FASD and FASD + NSC group were used in this study. Alterations of behavioral performance and brain neural circuit were evaluated 90 days after NSC treatment. (b) Changes of memory recognition function by prenatal ethanol exposure and NSC treatment in novel object recognition test. The investigation time to 'known' object and 'new' one were measured for control, FASD model (FASD) and FASD with NSC treatment (FASD + NSC) rats. Control rats investigated novel objects for a longer time than known objects, whereas FASD rats investigate both objects for a similar time $\left({ }^{*} P<0.05, n=7-9\right)$. In contrast, NSC treatment of FASD rats reversed this impairment, which showed a significantly longer time investigating novel objects ( ${ }^{*} P<0.05, n=9$ ). i.v., intravenous. p.o., oral administration. 
was dissected and trimmed in ice-cold Hank's balanced salt solution. Cells were dissociated by mechanical trituration and collected by centrifugation $\left(300 \mathrm{~g}\right.$ for $5 \mathrm{~min}$ at $4{ }^{\circ} \mathrm{C}$ ). The dissociated cells were plated in culture dishes coated with poly-L-ornithine/fibronectin in hneurobasal medium supplemented with $2 \%$ B27, $0.5 \mathrm{~mm}$ L-glutamine and $20 \mathrm{ng} \mathrm{ml}^{-1}$ fibroblast growth factor- 2 at a density of $5 \times 10^{4}$ cells cm ${ }^{-2}$ After 7 days of cell expansion culture under $5 \% \mathrm{CO}_{2}$ at $37{ }^{\circ} \mathrm{C}$, cells were stained with fluorescein-based dye to trace their migration by incubating the cells in phosphate-buffered saline buffer containing $5 \mu \mathrm{m}$ CFSE in constant agitation for $15 \mathrm{~min}$ at $37^{\circ} \mathrm{C}$, before administration.

NSC administration procedures. Although stem cell treatment has potential benefits for severe incurable neuropsychiatric disorders, there may be direct risks including tumor formation, immune rejection of administered stem cells, hemorrhage during neurosurgery and postoperative infection. ${ }^{16}$ To minimize invasiveness and risk of tumor formation by the NSC treatment, we chose intravenous administration. The tumor risk outcome depends on the degree of histocompatibility of the cells and circumstances (cell number, injection site, disease model, and so on) and a very low risk of teratomas was reported in NSC treatment studies using intravenous administration. ${ }^{17}$

At P60 after birth, a NSC suspension $\left(2 \times 10^{6}\right)$ mixed with $0.02 \%$ atelocollagen dissolved in saline in $0.5 \mathrm{ml}$ total fluid volume was injected slowly into the rats tail vein over $1 \mathrm{~min}$. We have established that $<0.02 \%$ of atelocollagen does not influence any NSC functions, including proliferation, migration, differentiation and survival (in submission). Although further investigation is needed to analyze the influence of intravenous injection of atelocollagen alone in vivo, we used saline as a vehicle for control. All rats received cyclosporine orally $\left(0.2 \mathrm{mg} \mathrm{ml}^{-1}\right.$ in their drinking water, corresponding to a blood concentration of $15 \mathrm{mg} \mathrm{kg}^{-1}$ daily intraperitoneally). ${ }^{18}$

Memory and social recognition testing. A novel object recognition test was performed according to a published method. ${ }^{19}$ In the habituation phase, rats were placed into an open-field apparatus consisting of a rectangular area ( $100 \mathrm{~cm}$ wide $\times 100 \mathrm{~cm}$ long $\times 60 \mathrm{~cm}$ high) made from acryl and allowed to adapt without objects for 15 min per day for 2 days for each rat. Then, during the training phase, two identical objects were presented to each rat for $10 \mathrm{~min}$. Twenty-four hours after the training phase, one of the old objects was replaced with a novel object and presented to each rat for $5 \mathrm{~min}$. To control the odor cues, the open-field arena and the objects were thoroughly cleaned with water, dried and ventilated for a few minutes between rats. Object exploration time was recorded using a video-assisted tracking system (Muromachi Kikai, Chuo-Ku, Tokyo, Japan). Discrimination between two objects was calculated using an investigation time counted over a 5-min span. ${ }^{19,20}$

Social recognition test was performed according to a recently published method ${ }^{21}$ with some modifications. In the habituation phase, rats were placed into an open-field apparatus the same as used in the novel recognition test, and allowed to adapt for 15 min per day for 7-10 days for each rat. Testing began when a stimulus female rat was introduced into the open-field of each male rat for a 1-min confrontation. At the end of the 1-min trial, we removed the stimulus animal and returned it to an individual holding cage. We repeated this sequence for four trials with 10-min inter-trial intervals and introduced each stimulus to the same male resident in all four trials unless otherwise indicated. In a fifth dishabituation trial, we introduced each stimulus rat to a different resident female rat. Behavior was recorded using a video-assisted tracking system (Muromachi Kikai), and scored by two trained raters.

Immunohistochemistry and PV-positive cell counts. After completing behavioral testing, rats were deeply anesthetized with isoflurane and transcardially perfused with heparinized saline $(0.5 \%)$ followed by $4 \%$ paraformaldehyde in phosphate buffer. The brains were then immersed in $4 \%$ paraformaldehyde, paraffin embedded and cut serially at the coronal plane into $20 \mu \mathrm{m}$ sections. A part of the intravenous administered NSCs that could migrate into brain were identified as GABAergic interneurons by double imaging of fluorescent CFSE dye labeling and immunostaining of GAD67 in the same visual field in the anterior cingulate cortex, hippocampus and amygdala. Briefly, sections were immunostained for GAD67 (rabbit anti-GAD67, 1:30; Santa Cruz, CA, USA) detected with Avidin and Biotinylated Complex (ABC) system (45 min, Vectastain ABC kit, Elite PK-6100, Vector Laboratories) and visualized with 3,3'diaminobenzidine (SK-4100, Vector Laboratories, Burlingame, CA, USA), followed by the detection of double-labeled cells with fluorescent dye and 3,3'-diaminobenzidine.

To obtain accurate and statistically significant counts of PV-positive interneurons, we performed immunohistochemistry using an antibody for PV (mouse anti-PV, 1:2000; Sigma, St Louis, MO, USA) detected with ABC system visualized with 3,3'-diaminobenzidine. Three pairs of animals in control, prenatal ethanol and prenatal ethanol + NSC treatment groups were examined. PV-positive cells were counted within a standardized rectangular area of $0.574 \mathrm{~mm}^{2}$ in the anterior cingulate cortex and amygdala, and $3.533 \mathrm{~mm}^{2}$ in hippocampus. Counts were performed in at least six slices selected at the same level for each animal. Sections were mounted with fluorescent mounting solution (Dako, Glostrup, Denmark), covered with a cover slide and sealed. Digital images were obtained with a fluorescence or optical instrument, Olympus BX52TF microscope (Olympus,Tokyo, Japan).

Western blot. After behavioral test, some of the animals from each group were deeply anesthetized with isoflurane and immediately thereafter, crude dissection of the brains was conducted and the anterior cingulated cortex, hippocampus and amygdala tissues were punched out and cryopreserved in $-80^{\circ} \mathrm{C}$. Subsequently, tissue was thawed and total protein was produced using RIPA Lysis Buffer (Santa Cruz). Protein concentration was determined using a BCA kit (Themo Scientific, Rockford, IL, USA). Protein sample underwent western blot analysis as described previously. ${ }^{22}$ Briefly, $20 \mu \mathrm{g}$ aliquots were subjected to sodium dodecyl sulfate-polyacrylamide gel electrophoresis on $10 \%$ polyacrylamide gels and transferred to polyvinylidene difluoride membranes. After blocking with $5 \%$ nonfat dry milk in Tris-buffered saline containing $0.05 \%$ Tween-20 overnight at 
$4{ }^{\circ} \mathrm{C}$, blots were probed with anti-postsynaptic density protein 95 (PSD-95) (rabbit, 1:1000; Cell Signaling Technology, Danvers, MA, USA) and glyceraldehyde 3-phosphate dehydrogenase (rabbit, 1:200; Santa Cruz) for $1.5 \mathrm{~h}$ at room temperature, then washed and incubated for $1 \mathrm{~h}$ with horseradish peroxidase-conjugated anti-rabbit immunoglobulin G (1:2000; Dako Cytomation, Glostrup, Denmark) respectively. Immunoreactive bands were detected with an enhanced chemiluminescence system (ECL system; GE Healthcare, Waukesha, WI, USA), and quantitatively analyzed by capturing images using a Sensobation Camera (UVP, Upland, CA, USA) in conjunction with the software, Vision WorksLS ver. 6.1.1 (UVP).

Statistical analysis. One-way analysis of variance and Tukey's HSD post hoc comparison were used to determine statistical significance, which was set at $P$ for all experimental observations. All values included in the figure legends represent mean \pm s.e.m. Statistical analyses were performed using SPSS 11.0 for Windows (SPSS Japan, Tokyo, Japan).

\section{Results}

Memory and cognitive impairment induced by fetal alcohol exposure. Three months after the administration of NSCs, controls and alcohol exposed rats were tested for their memory and cognitive behavior by measuring their investigation time for known and novel objects in a novel object recognition task (Figure 1a).

Control rats investigated novel objects for about twice as long as known objects, whereas FASD model rats investigated for almost the same amount of time the administration of NSCs reversed this memory and cognitive impairment, increasing the novel object investigating time, which was significantly longer than for known objects (Figure 1b).

Social recognition deficit induced by fetal alcohol exposure. In addition to a cognitive dysfunction, socioemotional deficits are a prominent neurobehavioral abnormality of FASD. ${ }^{23,24}$ To examine the effect of NSC treatment on socioemotional recognition decline, all male rats were tested for their social interaction behavior with a conspecific female. The social recognition by male rats manifests as a consistent decrease in olfactory investigation during repeated or prolonged encounters with a conspecific female. ${ }^{21,25}$ Control male rats showed a decline in the investigating time for a female on subsequent presentations of the same female in trials $2-4$, as compared with trial 1 (Figure 2a). This decrease was not due to a general decline in olfactory investigation, because presentation of a novel female during trial 5 resulted in a similar amount of investigation as trial 1 with the original female. Although FASD males showed sustained high levels of investigation at each encounter with the same female (Figure 2a). The investigation times at 1-4 trials were almost equal at trial 5 , in presenting with new females (Figure 2b). NSC treatment reversed this social recognition impairment, decreasing investigation time at trials 3 and 4 and increasing at trial 5 so that it was close to that of control rats. The NSC administration in control rats did not significantly affect investigation time (Figure $2 b$ ).

Detection of administered NSCs in the brain. Although we could not confirm complete absence of tumorigenic stem cells histochemically, intravenous administration of NSCs to FASD model rats as we performed did not produce any teratoma formation and growth throughout the experiments.

Ease of treatment and clinical precedent ${ }^{26,27}$ render intravenous administration desirable but several studies have reported poor cell delivery to the brain because of the bloodbrain barriers and suggested cell entrapment in peripheral organs. ${ }^{28}$ To reduce immune rejection and increase entry of administrated NSCs into brain, we pre-treated NSCs with atelocollagen a recently developed means of promoting gene delivery and achieved about a twofold higher migrating ratio of NSCs into brain in response to intravenous administration in FASD model rats (in submission). Prenatal ethanol exposure specifically reduces the density of the cortical GABAergic population in rodents. ${ }^{29}$ Therefore, to achieve regeneration of GABAergic neuronal circuits, which are assumed to be
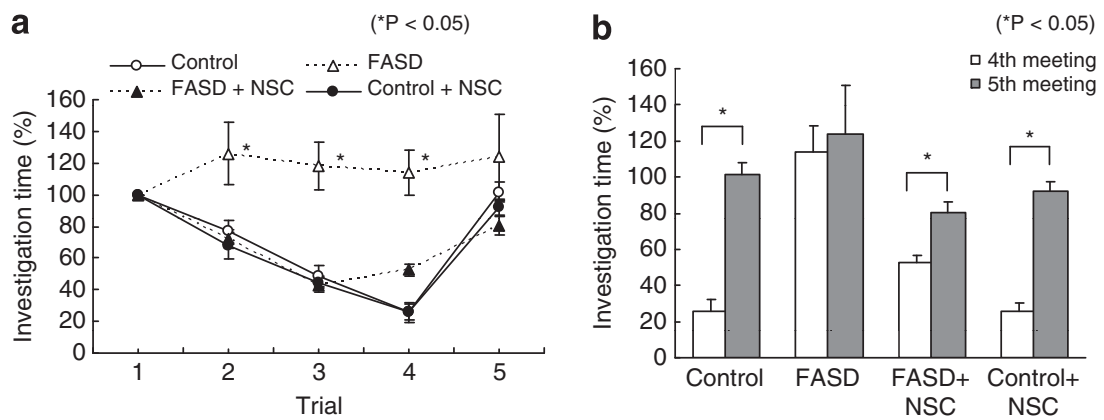

Figure 2 Neural stem cell (NSC) treatment reverses social recognition deficit in fetal alcohol spectrum disorder (FASD) model rats. Social recognition by male rats was measured as a difference in anogenital investigation. Data represent mean \pm s.e. $m$. for the amount of time allocated to investigating the same female during each of four 1 -min trials. A fifth dishabituation trial represents the response of males to the presentation of a new female in a 1-min pairing 10 min after the fourth trial ( $n=7-9)$. (a) Investigation time (\% amount of each first trial) allocated to investigation of control (open circle), FASD (open triangle), FASD + NSC (black triangle) and control + NSC (black circle) male rats. FASD rats showed significant increase of investigation time in $2-4$ th trials compared with controls ( $\left.{ }^{*} P<0.05\right)$. (b) Social recognition deficit and its correction by NSC treatment. Mean duration (\% amount of each first trial) of investigation of male rats in the fifth trial was significantly longer than fourth trial in FASD + NSC rats as well as control and control $+\mathrm{NSC}$ rats $\left({ }^{*} P<0.05\right)$. 

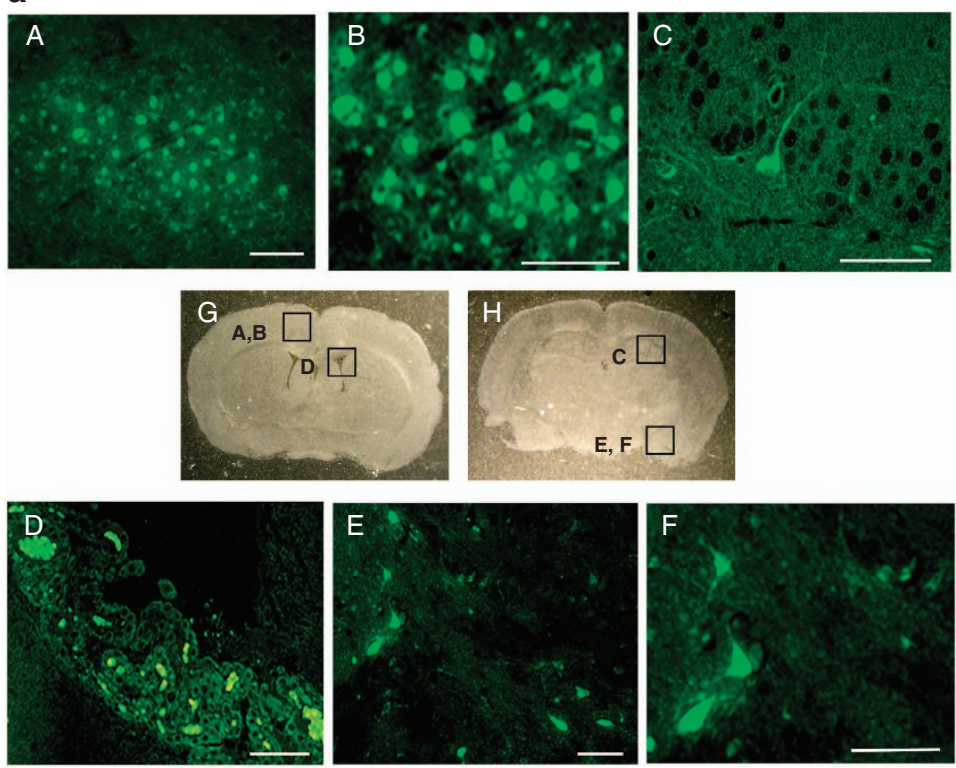

Figure 3 (a) Identification of migrated neural stem cells (NSCs) in the brain. Intravenously administered carboxyfluorescein diacetate succinimidyl ester (CFSE)-labeled NSCs detected in wide areas of the brain involving (A, B) anterior cingulate cortex, (C) hippocampus, (D) choroid plexus and (E, F) amygdala in fetal alcohol spectrum disorder (FASD) model rats by fluorescent images. (G) Coronal sections of anterior side (bregma A $1.00 \mathrm{~mm}$ ), and (H) posterior side (bregma P2.20). Scale bars: $100 \mu \mathrm{m}$ in $A, B, D, E$; $50 \mu \mathrm{m}$ in C, F. (b) Identification of administered NSCs as GABAergic cell. (A) Fluorescent images show administered cells (CFSE in green) in anterior cingulate cortex, hippocampus and amygdala. (B) Same cells were immunostained with GABAergic neuron marker (glutamic-acid-decarboxylase 67 (GAD67) in brown) in light photomicroscopic images.

impaired in FASD model rats, we prepared fetal rat telencephalon-derived NSCs for treatment by monolayer cultures, a method that generates GABAergic neurons efficiently. ${ }^{30}$ To analyze the potential migration of NCSs into brain, we labeled NSCs with CFSE, a fluorescent staining dye that can label all NSCs regardless of their differentiation. ${ }^{31} \mathrm{We}$ could detect CFSE-positive cells in several areas of FASD rat brain at 3-4 month after treatment (Figure 3a), and these marked cells also distributed in control rat brain (data not shown). A high proportion of administered cells is expected to differentiate into GABAergic neuron phenotype because of the culture method in this study. Indeed, a part of administered cells were identified as GAD67 containing cell in anterior cingulated cortex, hippocampus and amygdala (Figure $3 b$ ), which is reported specifically reduced in cortical PV-interneuron axon terminals in schizophrenia. ${ }^{12}$ We had previously demonstrated that intravenously administered $\left[{ }^{35} \mathrm{~S}\right]$-methionine-labeled NSCs survived and distributed throughout brains in FASD at 4 month after treatment, and greater radioactivities were counted in cortex, hippocampus, striatum and subventricular zone in FASD rat brains than those in controls. ${ }^{14}$ A particularly interesting question about the distribution difference between control and FASD brain needs to be analyzed precisely by using techniques, such as flow cytometry. These results indicated that intravenously administered NSCs could migrate across blood-brain barrier and delivered to cortical and limbic areas in the brain and survive at least for 4 month. Molecular mechanisms of NSCs crossing the blood-brain barrier is uncertain, but some hypothesizes exist including implications of direct effect of NSCs on bloodbrain barrier properties, ${ }^{32}$ and microchimerism of fetal brainderived NSCs. ${ }^{33}$ b
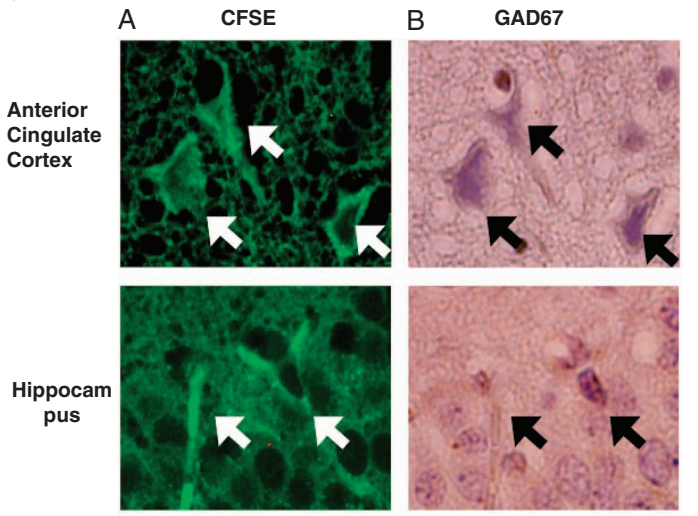

Amygdala
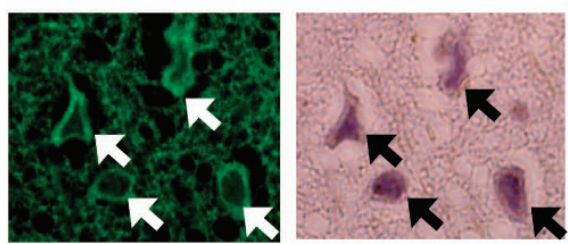

Alterations of PV-positive interneuron densities. Next, we examined whether GABAergic interneuron, especially PV-positive cells known to correlate with cognition and executive dysfunctions in schizophrenia and autism ${ }^{6,7}$ are altered in FASD model and NSC treatment. We discovered that, in FASD model rats, the number of PV-positive interneuron was decreased in anterior cingulated cortex, hippocampus and amygdala (Figures $4 \mathrm{a}$ and b). In contrast, treatment of NSCs significantly reversed these reductions in anterior cingulate cortex and amygdala but not in hippocampus (Figures $4 a$ and b). These results implicate the upregulation of PV-positive interneurons in anterior cingulate cortex and amygdala in the memory/social recognition recovery effect of NSC treatment. The data that PV-positive cell density in hippocampus were not changed by NSC treatment may relate to the lack of robust effect of this treatment on behavioral recovery in novel object recognition test than that in social interaction test.

Alterations of PSD-95 protein levels. To investigate the mechanisms underlying these lasting changes in GABAergic interneuron density, particularly PV-positive cell survival/generation, we hypothesized that synaptic remodeling may be associated with the pathophysiology of FASD and activity in NSC treatment. We measured protein expression levels of PSD-95 in the anterior cingulate cortex, hippocampus and amygdala of control and fetal ethanol exposure rats with and without subsequent treatment with NSCs. We observed significant changes of expression in anterior cingulate cortex and amygdala. PSD-95 protein levels were decreased in FASD rats, and reversed by NSC treatment (Figure 5). Although in hippocampus, the 
a
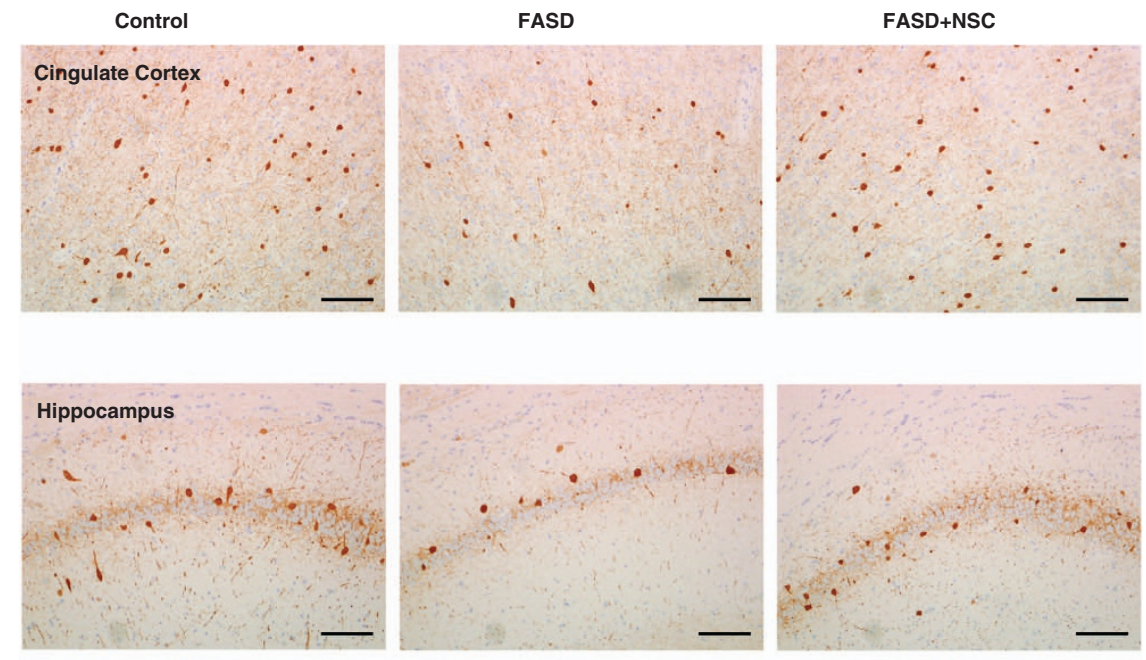

FASD+NSC
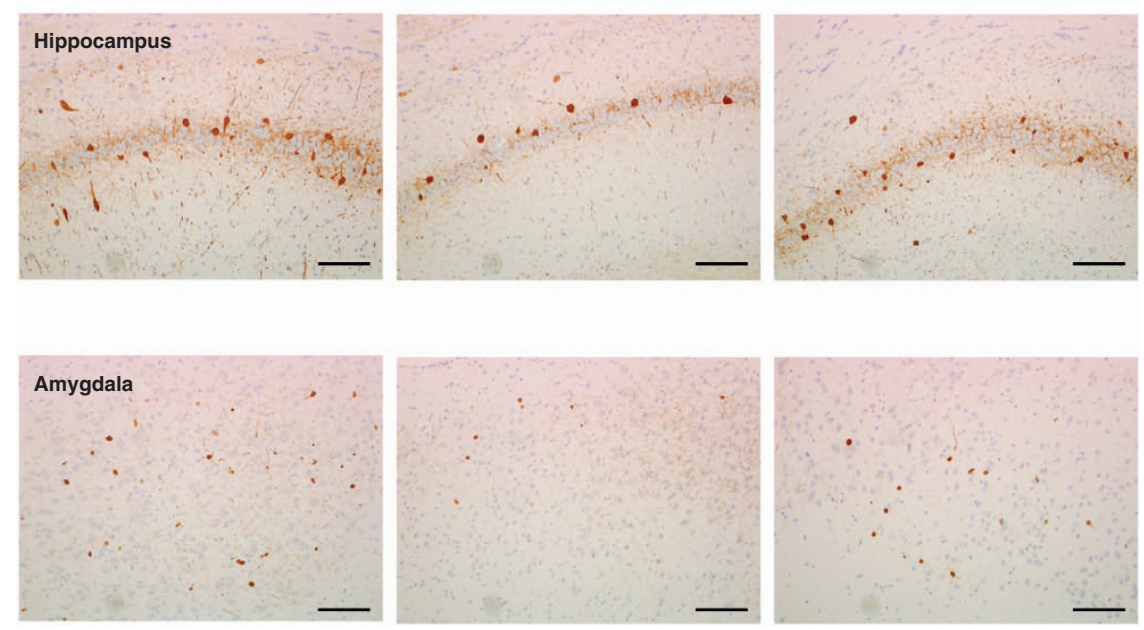

b

b $\quad\left({ }^{*} \mathrm{P}<0.05\right)$
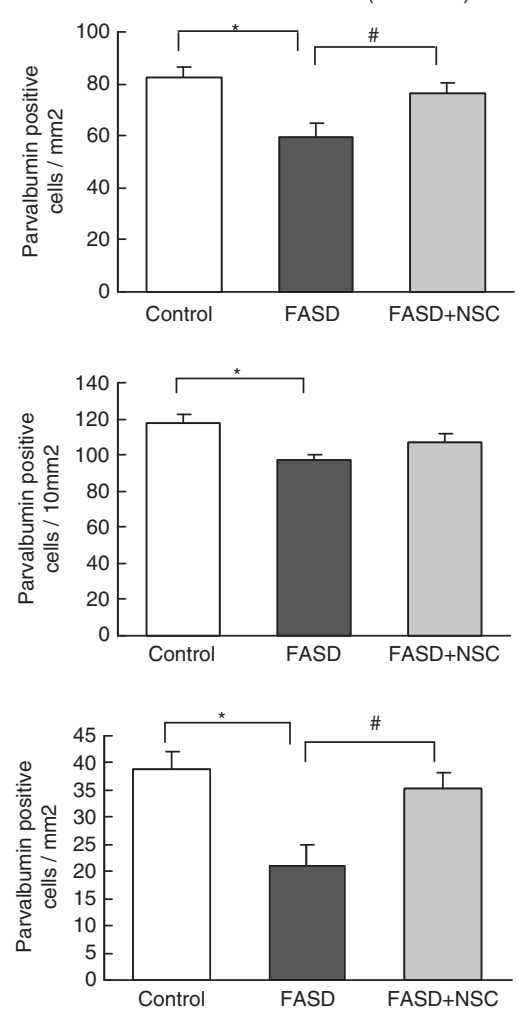

Figure 4 Decreased number of parvalbumin (PV)-positive interneurons and their area-dependent reverses by neural stem cell (NSC) treatment. (a) PV-positive cells were counted in coronal sections from control, fetal alcohol spectrum disorder (FASD) and FASD + NSC rats $(n=3)$. (b) Immunohistochemistry with $3,3^{\prime}$-diaminobenzidine (DAB)labeled anti-PV antibody indicated that the amount of PV-positive cells significantly decreased in anterior cingulate cortex, hippocampus and amygdala in FASD rats $\left({ }^{*} P<0.05\right)$. Although NSC treatment reversed these reductions in anterior cingulate cortex and amygdala $\left({ }^{\#} P<0.05\right)$. In contrast, NSC treatment did not show any influence on PV-positive cell amount change in hippocampus. Scale bars: $100 \mu \mathrm{m}$.

a Anterior Cingulate Cortex PSD-95

\section{$-m-m-m-m$}

GAPDH
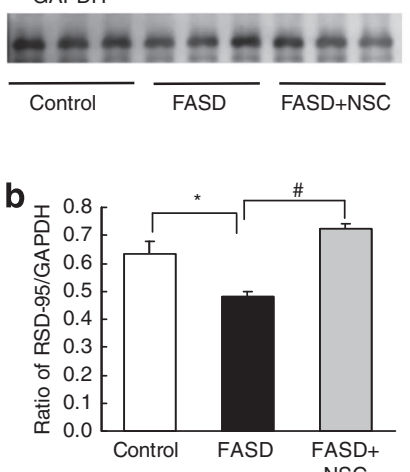

Hippocampus

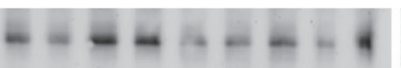

$-m-m-m-m$
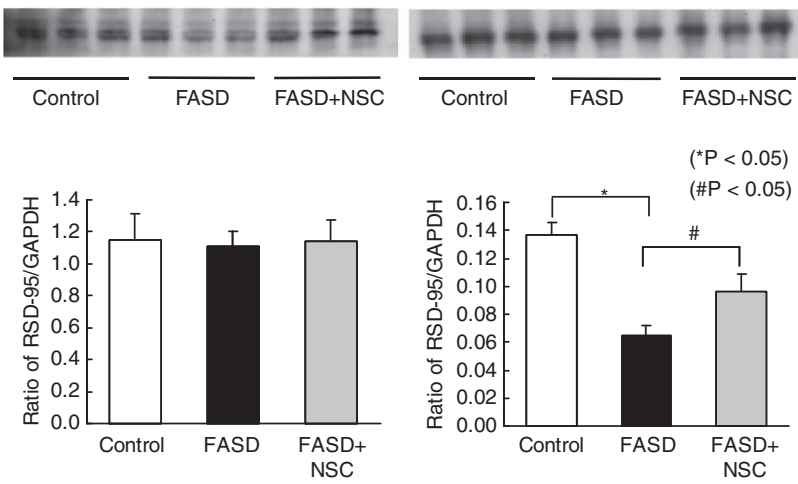

Figure 5 Decreased synaptogenesis in fetal alcohol spectrum disorder (FASD) model and their reverse by neural stem cell (NSC) treatment. (a) Amount of synapses was represented by postsynaptic density protein 95 (PSD-95) protein levels in western blot analysis. (b) Values of PSD-95 protein levels normalized to glyceraldehyde 3-phosphate dehydrogenase (GAPDH) were calculated in lysates from control, FASD and FASD + NSC rats $(n=4-6)$. PSD-95 protein levels significantly decreased in anterior cingulate cortex and amygdala in FASD rats $\left({ }^{\star} P<0.05\right)$. Although NSC treatment reversed these reductions in both areas $\left({ }^{\#} P<0.05\right)$. In contrast, in hippocampus, PSD-95 protein levels did not change in FASD and FASD + NSC rats. 
protein levels were unchanged in FASD and by NSC treatment (Figure 5).

\section{Discussion}

Intracerebral injection of NSCs is currently the most commonly used method to examine restoration of functional circuits in laboratory models of neurodegenerative pathologies. ${ }^{34,35}$ Although this method can produce direct recovery of damaged neural circuits, intraparenchymal injections into induced brain lesions is not an appropriate way to address diffuse or undefined cerebral pathologies in psychiatric illness, such as depression, schizophrenia and developmental disorders. The recent introduction of intravenous NSC administration overcomes this limitation in that cells can reach the cerebral parenchyma and induce recovery in models of multiple sclerosis, ${ }^{36}$ Huntington's disease ${ }^{37}$ and stroke. ${ }^{38}$ Another advantage is that they can reduce the risk of tumor formation, invasion and social debate about starting new trials of regenerative medicine in psychiatric disorders. Nevertheless, medical treatments using fetal brain-derived NSCs or embryonic stem cells raise ethical dilemmas. ${ }^{16}$ Further work is needed to examine efficiency when harvesting recent potential cell sources, such as mesenchymal stem cells and induced pluripotent stem cells. ${ }^{39}$

Using this method, we found several behavioral abnormalities were potentially reversible in FASD rats, which paralleled the neural circuit modifications in the anterior cingulate cortex, hippocampus and amygdala at the level of specific GABAergic interneuron density and changes in synaptogenesis. We discovered that recognition memory and social function, as assessed by novel object recognition and social interaction tests, are impaired in prenatally ethanol exposed rats, and that both of them is significantly attenuated by intravenous administration of NSCs at a time point when neural circuit development is already completed. In addition, NSC treatment restored the reduced number of $\mathrm{PV}$-positive interneurons and protein levels of PSD-95 in anterior cingulate cortex and amygdala. These findings could have profound implications for the treatment of FASD. Medications by antidepressants, mood stabilizers and antipsychotics are often used and are effective in ameliorating limited symptoms of FASD but not in curing the disorder. This underscores the importance of a better understanding of the pathological features in the brain of FASD victims and the need for better therapeutic methods to modify these processes.

The embryonic GABAergic system is vulnerable to various agents, ${ }^{40}$ and prenatal ethanol exposure causes gross morphological changes in GABAergic cells during development. Here, we found that PV-positive interneurons are clearly reduced in the anterior cingulate cortex and amygdala of FASD rats. As PV-positive cortical interneurons seem to be especially vulnerable to this type of developmental stress, and associative disruption of these cells was reported in various mental disorders, such as schizophrenia and autism, these findings indicate that loss of cortical and limbic PV-positive interneurons might also have a pivotal role in the pathophysiology of FASD. In addition, we observed that levels of PSD95 were decreased in the anterior cingulate cortex and amygdala in FASD. Recent findings suggest a new role for
PSD-95 in excitatory synapse development through a stabilizing effect by neuregulin 1 and its receptor ErbB4, which is specific to PV-positive GABAergic interneurons. ${ }^{41}$ Therefore, our data showing PSD-95 level changes may be paralleled with the results of PV-positive cell reductions in these areas. Although the cellular and molecular mechanisms of prenatal ethanol exposure-induced reduction of PV-positive neurons are unclear, recent findings suggest that ethanolinduced $\mathrm{GABA}_{\mathrm{A}}$ activation depolarizes NSCs, which causes transient intracellular $\mathrm{Ca}^{2+}$ increases, and reduces the number of proliferative stem cells during both embryonic development and adult neurogenesis. ${ }^{42,43}$ Another possibility involves impaired migration of interneurons, ${ }^{42}$ inhibition of neuronal differentiation ${ }^{15}$ and maturation ${ }^{44}$ caused by changes of brain-derived neurotrophic factor, which is considered to regulate both PV and GAD67 mRNA expression. ${ }^{45}$ As the mechanisms underlying impaired recognition and memory, abnormal synchronized oscillatory activity of principal cortical neurons has been focused on schizophrenia. As the ability of cortical PV-positive fast-spiking interneurons to drive synchronous oscillatory activity at gamma-frequency is being acknowledged as a cellular basis for cognitive and executive brain function, ${ }^{46}$ it is plausible that a reduction of PV-positive interneurons in the anterior cingulate cortex in FASD rats could correlate with the impaired memory and recognition as assessed by the novel object recognition test. However, further research is needed to understand the mechanisms of cognitive dysfunction of FASD, especially the implications for other sub-populations of GABAergic interneurons including somatostatin- and neuropeptide $\mathrm{Y}$-positive neurons.

PV-positive interneuron reduction and PSD-95 decrease were most evident in the amygdala in the FASD model compared with controls. Although studies about GABAergic interneuron dysfunction in amygdala is incomplete, many researchers have suggested that disruption of the amygdala function results in disturbance of social interaction, ${ }^{47,48}$ In our behavioral experiment, FASD model rats showed strong abnormalities in social recognition tests, and similar social recognition deficit was reported in CD38 knockout autism model mice. ${ }^{21}$ Recently, chronic administration of phencyclidine was shown to cause disturbed social behavior, which was alleviated by infusion of oxytocin into the amygdala. ${ }^{48} \mathrm{PV}$ positive interneuron loss in lateral amygdala was suggested in maternal deprivation stress exposed rats. ${ }^{47}$ As social/emotional problems are one of the major symptoms of FASD, the results of PV-positive interneuron loss in amygdala may represent an important neural circuit disruption related to pathophysiology of FASD.

Notable findings of this study are that intravenous NSCs enveloped with atelocollagen could restore reductions in PVpositive interneurons in the anterior cingulated cortex and amygdala, and was associated with recovery of reduced PSD95 levels in the FASD model. Furthermore, NSC treatment utilizing atelocollagen displayed potential therapeutic efficacy against memory and social recognition deficits demonstrated in this psychiatric model. Although the mechanism underlying these effects of NSC treatment cannot be easily explained, there may be several possibilities accounting for their beneficial actions. One is a neuroprotective effect exerted 
by the administered NSCs. Recently reported is that administration of olfactory ensheathing cells into the dorsal hemisected spinal cord results in improved functional outcome and elevation in the brain-derived neurotrophic factor levels. ${ }^{49}$ Another possibility is upregulation of neurogenesis. New generation and migration from precursors in subventricular zone or NG2 + cells within the cortex might facilitate the trophic effect of administered NSCs. ${ }^{50,51}$ Another possible mechanism is that administered NSCs integrate into tissue and replace damaged cells. Our immunohistochemical data suggest that a proportion of intravenously administered CFSE-labeled NSCs were located in the anterior cingulate cortex, hippocampus and amygdala, including the area where PV-positive neuron reverse occurred by the treatment. Some of the CFSE-labeled NSCs showed GAD67 positivity, the protein known to be involved in the axon terminals of PVinterneurons. ${ }^{11}$ In a previous study, we have investigated the peripherally injected cell migration and distribution in the brain by using double labeling with CFSE and [ $\left.{ }^{35} \mathrm{~S}\right]$-methionine. By this method, we can surely perform quantitative analysis. ${ }^{14}$ However, because of the strong aims to investigate neurohistochemical and molecular level analysis, we chose the method for labeling the cells only with CFSE-dye in this study. Further investigation is needed to clearly characterize the full potential of administered NSC to differentiate into GABAergic interneurons, and their possible contribution to behavioral recoveries. Further investigation is needed to clearly characterize the full potential of administered NSC to differentiate into GABAergic interneurons, and their possible contribution to behavioral recoveries.

In the hippocampus, prenatal ethanol exposure induced a PV-positive interneuron loss that was smaller than anterior cingulated cortex and amygdala, and NSC treatment did not affect this change. It is known that influence of prenatal ethanol exposure depends on the period of development. A recent report demonstrated that terminal deoxynucleotidyl transferase-mediated dUTP nick end labeling analysis of E11-E14 ethanol-treated embryos (similar to our protocol: E10-E13) at E14 showed a significant increase in apoptotic cells in the cortex, but not in the hippocampus, which suggests a region specific or a developmentally related cell death effect. ${ }^{9}$ The hippocampal PSD-95 protein level change was apparently different from those in other areas. PSD-95 was not changed by prenatal ethanol exposure or NSC treatment. It can be considered that PSD-95 is known as a synaptic protein contained in the glutamatergic neurons, therefore, the data may show the result of the predominant state of excitatory cells in hippocampus caused by ethanol treatment. $^{52}$

We view the PV-positive interneuron as a prototypical example of how prenatal ethanol exposure and NSC treatment produce neural circuit and behavioral changes. Although many questions remain about the mechanisms by which fetal alcohol exposure and NSC treatment cause changes in the GABAergic neuron system, the results of this study provide fundamentally new information concerning the detailed molecular/cellular mechanisms underlying deleterious effects of prenatal ethanol exposure on the brain and their potential reversal by intravenous treatment with NSCs. More generally, our results provide further support for the notion that intravenous treatment of NSCs complexed with atelocollagen is a potential therapeutic method, which should be tested in incurable treatmentresistant psychiatric disorders.

\section{Conflict of interest}

The authors declare no conflict of interest.

Acknowledgements. Funding for this study was provided by grant-in-aid for Science Research No. 21390334 (Toshikazu Saito) and No. 22791132 (Tomohiro Shirasaka) from Japan Society for the Promotion of Science (JSPS). The funders had no further role in study design, or in the collection, analysis and interpretation of data; in writing the report, or in the decision to submit the paper for publication. We thank Dr Peter M Olley (Sapporo Medical University) for pertinent language advice. We are thankful to Dr Tadafumi Kato (Brain Science Institute, RIKEN) for his invaluable assistance and discussion on this study.

Author contributions. EH and TS designed the study and WU wrote the protocol. TY and MT managed the literature searches and analyses. WU undertook the statistical analysis and EH and TI supported the experiments technically. EH and WU wrote the first draft of the manuscript. All authors contributed to and have approved the final manuscript.

1. Riley EP, McGee CL. Fetal alcohol spectrum disorders: an overview with emphasis on changes in brain and behavior. Exp Biol Med (Maywood) 2005; 230: 357-365.

2. Mattson SN, Riley EP. A review of the neurobehavioral deficits in children with fetal alcohol syndrome or prenatal exposure to alcohol. Alcohol Clin Exp Res 1998; 22: 279-294.

3. Archibald SL, Fennema-Notestine C, Gamst A, Riley EP, Mattson SN, Jernigan TL. Brain dysmorphology in individuals with severe prenatal alcohol exposure. Dev Med Child Neurol 2001; 43: 148-154.

4. Mattson SN, Goodman AM, Caine C, Delis DC, Riley EP. Executive functioning in children with heavy prenatal alcohol exposure. Alcohol Clin Exp Res 1999; 23: 1808-1815.

5. Miller MW. Mechanisms of ethanol induced neuronal death during development: from the molecule to behavior. Alcohol Clin Exp Res 1996; 20: 128A-132A.

6. Miller MW. Effect of prenatal exposure to ethanol on glutamate and GABA immunoreactivity in macaque somatosensory and motor cortices: critical timing of exposure. Neuroscience 2006; 138: 97-107.

7. Guidotti A, Auta J, Davis JM, Dong E, Grayson DR, Veldic M et al. GABAergic dysfunction in schizophrenia: new treatment strategies on the horizon. Psychopharmacology (Berl) 2005; 180: 191-205

8. Belmonte MK, Cook EH Jr., Anderson GM, Rubenstein JL, Greenough WT, BeckelMitchener $\mathrm{A}$ et al. Autism as a disorder of neural information processing: directions for research and targets for therapy. Mol Psychiatry 2004; 9: 646-663.

9. Isayama RN, Leite PE, Lima JP, Uziel D, Yamasaki EN. Impact of ethanol on the developing GABAergic system. Anat Rec (Hoboken) 2009; 292: 1922-1939.

10. Akbarian S, Huang HS. Molecular and cellular mechanisms of altered GAD1/GAD67 expression in schizophrenia and related disorders. Brain Res Rev 2006; 52: 293-304.

11. Curley AA, Arion D, Volk DW, Asafu-Adjei JK, Sampson AR, Fish KN et al. Cortical deficits of glutamic acid decarboxylase 67 expression in schizophrenia: clinical, protein, and cell type-specific features. Am J Psychiatry 2011; 168: 921-929.

12. Gonzalez-Burgos G, Hashimoto T, Lewis DA. Alterations of cortical GABA neurons and network oscillations in schizophrenia. Curr Psychiatry Rep 2010; 12: 335-344.

13. Nakazawa K, Zsiros V, Jiang Z, Nakao K, Kolata S, Zhang S et al. GABAergic interneuron origin of schizophrenia pathophysiology. Neuropharmacology 2011; 62: 1574-1583.

14. Yoshinaga T, Hashimoto E, Ukai W, Toki S, Saito S, Saito T. Neural stem cell transplantation in a model of fetal alcohol effects. J Neural Transm Suppl 2007 331-337.

15. Tateno M, Ukai W, Yamamoto M, Hashimoto E, Ikeda H, Saito T. The effect of ethanol on cell fate determination of neural stem cells. Alcohol Clin Exp Res 2005; 29: 225S-229S.

16. Master Z, McLeod M, Mendez I. Benefits, risks and ethical considerations in translation of stem cell research to clinical applications in Parkinson's disease. J Med Ethics 2007; 33: 169-173.

17. Dressel R. Effects of histocompatibility and host immune responses on the tumorigenicity of pluripotent stem cells. Semin Immunopathol 2011; 33: 573-591.

18. Barzilay R, Ben-Zur T, Sadan O, Bren Z, Taler M, Lev N et al. Intracerebral adult stem cells transplantation increases brain-derived neurotrophic factor levels and protects against phencyclidine-induced social deficit in mice. Translational Psychiatry 2011; 1: e61.

19. Dix SL, Aggleton JP. Extending the spontaneous preference test of recognition: evidence of object-location and object-context recognition. Behav Brain Res 1999; 99: 191-200.

20. Bevins RA, Besheer J. Object recognition in rats and mice: a one-trial non-matchingto-sample learning task to study 'recognition memory'. Nat Protoc 2006; 1: 1306-1311. 
21. Jin D, Liu HX, Hirai H, Torashima T, Nagai T, Lopatina $O$ et al. CD38 is critical for socia behaviour by regulating oxytocin secretion. Nature 2007; 446: 41-45.

22. Ishii T, Hashimoto E, Ukai W, Tateno M, Yoshinaga T, Saito S et al. Lithium-induced suppression of transcription repressor NRSF/REST: effects on the dysfunction of neuronal differentiation by ethanol. Eur J Pharmacol 2008; 593: 36-43.

23. Streissguth AP, Aase JM, Clarren SK, Randels SP, LaDue RA, Smith DF. Fetal alcoho syndrome in adolescents and adults. JAMA 1991; 265: 1961-1967.

24. Conry J. Neuropsychological deficits in fetal alcohol syndrome and fetal alcohol effects. Alcohol Clin Exp Res 1990; 14: 650-655.

25. Takayanagi Y, Yoshida M, Bielsky IF, Ross HE, Kawamata M, Onaka T et al. Pervasive social deficits, but normal parturition, in oxytocin receptor-deficient mice. Proc Natl Acad Sci USA 2005; 102: 16096-16101.

26. Sasaki M, Honmou O, Radtke C, Kocsis JD. Development of a middle cerebral artery occlusion model in the nonhuman primate and a safety study of i.v. infusion of human mesenchymal stem cells. PLoS One 2011; 6: e26577.

27. Mitrecic $D$. Current advances in intravascular administration of stem cells for neurologica diseases: a new dose of rejuvenation injected. Rejuvenation Res 2011; 14: 553-555.

28. Gao J, Dennis JE, Muzic RF, Lundberg M, Caplan Al. The dynamic in vivo distribution of bone marrow-derived mesenchymal stem cells after infusion. Cells Tissues Organs 2001; 169: 12-20.

29. Bailey CD, Brien JF, Reynolds JN. Chronic prenatal ethanol exposure alters the proportion of GABAergic neurons in layers II/III of the adult guinea pig somatosensory cortex. Neurotoxicol Teratol 2004; 26: 59-63.

30. Shin E, Palmer MJ, Li M, Fricker RA. GABAergic neurons from mouse embryonic stem cells possess functional properties of striatal neurons in vitro, and develop into striatal neurons in vivo in a mouse model of Huntington's disease. Stem Cell Rev 2011; 8: $513-531$

31. Wang PH, Schwindt TT, Barnabe GF, Motta FL, Semedo P, Beraldo FC et al. Administration of neural precursor cells ameliorates renal ischemia-reperfusion injury. Nephron Exp Nephrol 2009; 112: e20-e28.

32. Lim JC, Wolpaw AJ, Caldwell MA, Hladky SB, Barrand MA. Neural precursor cell influences on blood-brain barrier characteristics in rat brain endothelial cells. Brain Res 2007; 1159: 67-76.

33. Dawe GS, Tan XW, Xiao ZC. Cell migration from baby to mother. Cell Adh Migr 2007; 1 19-27.

34. Vitry S, Avellana-Adalid V, Lachapelle F, Evercooren AB. Migration and multipotentiality of PSA-NCAM + neural precursors transplanted in the developing brain. Mol Cell Neurosci 2001; 17: 983-1000

35. Fisher LJ. Neural precursor cells: applications for the study and repair of the centra nervous system. Neurobiol Dis 1997; 4: 1-22.

36. Pluchino S, Zanotti L, Rossi B, Brambilla E, Ottoboni L, Salani G et al. Neurospherederived multipotent precursors promote neuroprotection by an immunomodulatory mechanism. Nature 2005; 436: 266-271.

37. Lee ST, Chu K, Park JE, Lee K, Kang L, Kim SU et al. Intravenous administration of human neural stem cells induces functional recovery in Huntington's disease rat model. Neurosci Res 2005; 52: 243-249.

38. Honmou O, Houkin K, Matsunaga T, Niitsu Y, Ishiai S, Onodera R et al. Intravenous administration of auto serum-expanded autologous mesenchymal stem cells in stroke. Brain 2011; 134: 1790-1807.
39. Tsuji O, Miura K, Fujiyoshi K, Momoshima S, Nakamura M, Okano H. Cell therapy for spinal cord injury by neural stem/progenitor cells derived from iPS/ES cells. Neurotherapeutics 2011; 8: 668-676.

40. Cuzon VC, Yeh PW, Yanagawa Y, Obata K, Yeh HH. Ethanol consumption during early pregnancy alters the disposition of tangentially migrating GABAergic interneurons in the fetal cortex. J Neurosci 2008; 28: 1854-1864.

41. Ting AK, Chen $Y$, Wen L, Yin DM, Shen C, Tao $Y$ et al. Neuregulin 1 promotes excitatory synapse development and function in GABAergic interneurons. J Neurosci 2011; 31: $15-25$

42. Alcantara S, Pozas E, Ibanez CF, Soriano E. BDNF-modulated spatial organization of Cajal-Retzius and GABAergic neurons in the marginal zone plays a role in the development of cortical organization. Cereb Cortex 2006; 16: 487-499.

43. Liu X, Wang Q, Haydar TF, Bordey A, Nonsynaptic GABA. signaling in postnatal subventricular zone controls proliferation of GFAP-expressing progenitors. Nat Neurosci 2005: 8: 1179-1187.

44. Singh AK, Gupta S, Jiang Y, Younus M, Ramzan M. In vitro neurogenesis from neural progenitor cells isolated from the hippocampus region of the brain of adult rats exposed to ethanol during early development through their alcohol-drinking mothers. Alcohol Alcohol 2009; 44: 185-198.

45. Marty S, Berzaghi Mda P, Berninger B. Neurotrophins and activity-dependent plasticity of cortical interneurons. Trends Neurosci 1997; 20: 198-202.

46. Fries P. Neuronal gamma-band synchronization as a fundamental process in cortical computation. Annu Rev Neurosci 2009; 32: 209-224.

47. Giachino C, Canalia N, Capone F, Fasolo A, Alleva E, Riva MA et al. Maternal deprivation and early handling affect density of calcium binding protein-containing neurons in selected brain regions and emotional behavior in periadolescent rats. Neuroscience 2007; 145: 568-578.

48. Lee PR, Brady DL, Shapiro RA, Dorsa DM, Koenig JI. Social interaction deficits caused by chronic phencyclidine administration are reversed by oxytocin. Neuropsychopharmacology 2005; 30: 1883-1894

49. Sasaki M, Hains BC, Lankford KL, Waxman SG, Kocsis JD. Protection of corticospinal tract neurons after dorsal spinal cord transection and engraftment of olfactory ensheathing cells. Glia 2006; 53: 352-359.

50. Wang DD, Kriegstein AR. Defining the role of GABA in cortical development. J Physiol 2009; 587: 1873-1879.

51. Dayer AG, Cleaver KM, Abouantoun T, Cameron HA. New GABAergic interneurons in the adult neocortex and striatum are generated from different precursors. J Cell Biol 2005; 168 : 415-427.

52. Kim KC, Go HS, Bak HR, Choi CS, Choi I, Kim P et al. Prenatal exposure of ethano induces increased glutamatergic neuronal differentiation of neural progenitor cells. J Biomed Sci 2010; 17: 85

Translational Psychiatry is an open-access journal published by Nature Publishing Group. This work is licensed under the Creative Commons Attribution-NonCommercial-No Derivative Works 3.0 Unported License. To view a copy of this license, visit http://creativecommons.org/licenses/by-nc-nd/3.0/ 